\title{
Welcome to the 15th volume of Future Virology
}

\author{
Atiya Henry*,1 \\ ${ }^{1}$ Future Science Group, Unitec House, London N3 1QB, UK \\ *Author for correspondence: a.henry@futuremedicine.com
}

\begin{abstract}
Keywords: cytomegalovirus • endogenous retroviruses $\bullet$ hepatitis C virus • HIV • Mayaro virus • ribavirin • viral reservoir • virology
\end{abstract}

To our readers, we would like to wish you a very happy new year from all of us here at Future Medicine and introduce you to the 15th volume of Future Virology. I would like to begin 2020 with a thank you to our readers, esteemed editorial board, peer reviewers and contributing authors for giving their continued support. We look forward to working with you all in the forthcoming year. This foreword presents some of our content highlights from 2019 alongside some exciting future prospects for the journal, in the year ahead.

\section{Content highlights}

2019 was another successful year for Future Virology. The journal fulfilled its purpose by publishing exciting current research from the experts in the field, documenting the fast-evolving world of virology.

The top read article, at the time of writing (January 2020), was an editorial article entitled 'HIV cure strategies: a complex approach for a complicated viral reservoir?' written by Marsden and Zack [1]. In this piece the authors touch upon different strategies that could be used to tackle HIV latency, such as transplantation approaches and genome editing. They conclude that the key to a successful HIV cure may lie in using a combination of these different strategies.

Our second most read article was 'Mayaro virus: a neglected threat could cause the next worldwide viral epidemic' by de O Mota et al. [2]. This editorial article provides an update on the Mayaro virus, exploring its transmission cycle, recent cases and epidemiological findings.

A further highly popular article was a special report entitled 'Ribavirin: pharmacology, multiple modes of action and possible future perspectives', written by Nyström et al. [3]. Here, the authors give an overview of the pharmacology and proposed mode of action of Ribavirin, a guanosine analog with activity against RNA and DNA viruses. The piece ends with a look into the future, exploring potential novel uses of Ribavirin.

The final article to mention is an editorial authored by Bamford and McLauchlan. The piece entitled 'Comparative host genomics: how has human evolution affected our immune defence against hepatitis $C$ virus?' discusses how comparative genomics can unravel the molecular mechanisms behind outcome of hepatitis $\mathrm{C}$ infection [4]. The authors explain that by inoculating mammalian species with hepatitis $\mathrm{C}$, analyzing their whole genome sequences and comparing them to their human counterparts, we can identify which specific genetic changes have modulated our immune response to hepatitis $\mathrm{C}$ virus.

\section{Readership demographics}

It is always interesting to consider the demography of our readership, as it continues to change with the increasing growth of Future Virology. The year 2019 saw a 4\% increase in the proportion of readership in the USA, determining the USA as our biggest content reader. Our second and third biggest readership belonged to Asia (33\%) and Europe (20\%; Figure 1). We expect to see continued growth in emerging scientific communities, particularly in areas afflicted by viral outbreaks. We hope to continue our work toward reaching a truly globalized audience.

\section{Top Altmetric articles}

We have been working with Altmetric since 2015 to help visualize where the articles we publish are having an online impact:

Future Medicine 
Future Virology readership demographics

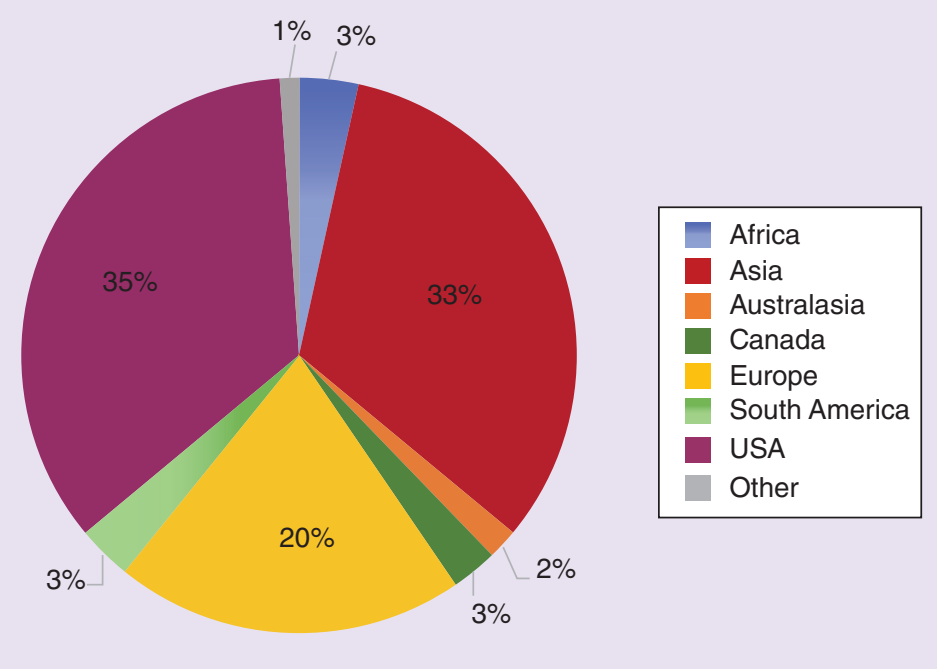

Figure 1. Future Virology readership demographic 2019.

- The Future Virology article with the highest Altmetic score in 2019 was the aforementioned editorial article entitled 'Comparative host genomics: how has human evolution affected our immune defense against hepatitis $C$ virus? [4] which was in the top $25 \%$ of all research outputs scored by Altmetric;

- Our second highest score was from a review piece entitled 'Catch me if you can: the arms race between human cytomegalovirus and the innate immune system' [5];

- Coming in third place was another review article: 'Emerging technologies for the detection of viral infections' [6].

\section{Social media}

Future Virology remains active across social media and you can stay up to date with the latest on Twitter (@fsgfvl) [7] and LinkedIn [8], where our following has continued to rise. Across these platforms we consider forthcoming research in the field of virology, as well as current news, journal highlights and newly published articles. Through these mediums we are able to enjoy engaging with our readers and experts in the field.

\section{Infectious Diseases Hub}

Future Virology is proudly partnered with Infectious Diseases Hub (ID Hub) [9], a free-access online resource that provides a platform to allow quick access to the latest, essential research to its members on all aspects of virology and microbiology, from the bench to the bedside. Breaking news, exclusive features, interviews, peer-reviewed articles and more are covered in ID Hub, provided by Future Science Group [10]. Furthermore, three articles from the current issue are nominated each month to feature on ID Hub, at the discretion of the editor. This affiliation has allowed the content of Future Virology to have wider global reach and increased dissemination and influence through a multitude of different platforms.

One standout example in 2019 was the Peek Behind the Paper feature on the emerging field of endogenous retroviruses with lead author, Enzo Tramontano [11,12]. ID Hub not only offered free access to the full article to all its members but also published an exclusive interview in which potential links between human endogenous retroviruses and disease are discussed, with an outlook to future directions [12].

\section{Conference attendance}

As always, members of the Future Virology department attended a number of conferences, meetings and events around the world in 2019, allowing us to meet our readers, authors, editorial board members and collaborators, as well as to keep up-to-date with the field and ensure full coverage of all the latest and ground-breaking research. In 2019, we attended the following events:

- Microbiology Society annual conference (8-11 April, Belfast, UK); 
- The European Congress of Clinical Microbiology and Infectious Diseases (ECCMID; 13-16 April, Amsterdam, The Netherlands);

- American Society for Microbiology (ASM) Microbe (20-24 June, San Francisco, CA, USA).

The Future Virology team looks forward to attending more conferences to continue the journal's development. To follow which events the team will attend in 2019, please follow the journal on Twitter [7], join our LinkedIn group [8] or become a member of ID Hub for free [9].

\section{Conclusion}

As always, any feedback that the virology community wishes to share is appreciated, whether this be recommendations of up-and-coming areas you think deserve coverage or advice on the future direction of our content, corresponding to changing directions in the field. We welcome unsolicited research, review, editorial and other opinion article proposals, among others, and would be delighted to hear from you if you are interested in submitting to the journal. We are especially interested in submissions covering key aspects of virology, including:

- The molecular basis of viral diseases;

- Virus-host interactions;

- New and re-emerging viruses;

- Epidemiologic studies and trends;

- Advances in oncolytic viral therapy, viral gene therapy and viral immunotherapy;

- Vaccines and antiviral agents;

- Adverse events and drug safety;

- The problem of viral drug resistance and potential methods to overcome this;

- Pharmacoeconomics and cost-benefit issues in virology;

- The use of viral vectors in gene therapy and functional genomics;

- Defense against the use of viruses in bio-terrorism;

- Real-world evidence and outcomes research.

Financial \& competing interests disclosure

The author is an employee of Future Science Group. The author has no other relevant affiliations or financial involvement with any organization or entity with a financial interest in or financial conflict with the subject matter or materials discussed in the manuscript apart from those disclosed.

No writing assistance was utilized in the production of this manuscript.

\section{References}

1. Marsden MD, Zack JA. HIV cure strategies: a complex approach for a complicated viral reservoir? Future Virol. 14(1), 5-8 (2019).

2. de O Mota MT, Avilla CM, Nogueira ML. Mayaro virus: a neglected threat could cause the next worldwide viral epidemic. Future Virol. 14(6), 375-377 (2019).

3. Nyström K, Waldenström J, Tang KW, Lagging M. Ribavirin: pharmacology, multiple modes of action and possible future perspectives. Future Virol. 14(3), 153-160 (2019).

4. Bamford CG, McLauchlan J. Comparative host genomics: how has human evolution affected our immune defence against hepatitis C virus? Future Virol. 14(3), 125-128 (2019).

5. Galitska G, Biolatti M, Griffante G et al. Catch me if you can: the arms race between human cytomegalovirus and the innate immune system. Future Virol. 14(4), 247-263 (2019).

6. Burbelo PD, Iadarola MJ, Chaturvedi A. Emerging technologies for the detection of viral infections. Future Virol. 14(1), 39-49 (2019).

7. Future Virol. Twitter. twitter.com/fsgfvl

8. Future Science Group Virology. LinkedIn. www.linkedin.com/groups/8506116

9. Infectious Diseases Hub. www.id-hub.com

10. Future Science Group. www.future-science-group.com/

11. Grandi N, Pisano MP, Tramontano E. The emerging field of human endogenous retroviruses: understanding their physiological role and contribution to diseases. Future Virol. 14(7), 441-444 (2019).

12. Tramontano E. A peek behind the paper - Enzo Tramontano on the emerging field of endogenous retroviruses. ID Hub (2019). www.id-hub.com/2019/10/03/peek-behind-paper-enzo-tramontano-emerging-field-endogenous-retroviruses/ 
